\title{
SEX AND GENDER IN HEALTH AND MEDICINE*
}

\author{
LOIS M. VERBRUGGE \\ Department of Biostatistics, School of Public Health, The University of Michigan, \\ Ann Arbor, MI 48109, U.S.A.
}

A principal theme of the Fifth International Conference on Social Science and Medicine was "Sex and Gender in Health and Medicine". Sex has important implications for individual health, and it is a prominent feature in the staffing and utilization of health services. Sex is an appropriate focus of research on social aspects of health and medicine: first, sex differences in morbidity, disability, and utilization of health services are pervasive in the world, but the causes of these differences are not fully understood. Second, there are large sex differences in the composition of health professions. What social filters explain strong differential recruitment by sex to these professions? Do male and female members of the same profession behave similarly? Third, most health care occurs outside the medical system. Women play an active role in non-market health services, providing care for sick family members and educating family members about health and health practices. Both sexes are involved in traditional medicine, but there are sex differences in the types of services they offer. Fourth, some health conditions are sex-specific (e.g. pregnancy, prostrate infection). Health services for reproduction (birth prevention, pregnancy, childbirth, and obstetric/gynecological problems) are designed primarily for women. Expaniding reproductive options and improving access to reproductive health services are important issues in many nations. Fifth, the interaction of patient and health professional prompts some intriguing questions. Does a patient's sex influence diagnoses and treatments rendered by physicians? How do patients react to a health. professional whose sex is "unusual" (e.g. male nurse, female surgeon)?

These topics differ in how much scientific and political fervor they have inspired. Problems of equal opportunities for professional training, reproductive options and services, and sex biases in physician treatment of patients have prompted active political discussion and sometimes legislation. Scientific research on these topics varies. There is a vast literature on reproduction, somewhat less on sex differences in professional training, and rather little on physician sex biases. The other topics named above have stimulated little political discussion. Sex differences in morbidity, utilization, and mortality are subjects of persistent research. Very little is known about non-market health services, or how men and women are involved in providing them. Similarly, little empirical research exists on the lay public's expectations about health professionals' sex, and their reactions to "unusual" practitioners.

* Report of Discussions for a Conference Theme, Fifth International Conference on Social Science and Medicine, Nairobi, Kenya, 8-12 August 1977.
The Discussion Group devoted its time to considering scientific hypotheses and evidence on the following topics:

(1) Sex differences in morbidity, disability, and health services utilization. ("Morbidity" refers to acute and chronic illness, injury, and impairments. "Disability" means temporary or permanent limitation in mobility, or in social activities. "Health services utilization" means contacts with modern medical care services.)

(2) Men and women as health professionals.

(3) The roles of men and women in providing nonmarket and traditional health services. ("Nonmarket" services are mainly home services, including health care for ill family members and health education for all members. "Traditional" services refer to diagnosis and treatment by medicine men, indigenous midwives, and other health professionals practicing outside the modern system.)

(4) Sex-specific health conditions, especially issues about contraception, abortion, pregnancy, obstetrics, and gynecology.

(5) Sex biases in medical care and in public expectations about medicine.

During the Conference, the Discussion Group focused on Items 1, 2 and 4. This emphasis reflected (a) the main interests and expertise of group members and (b) the topics of papers presented to the group for discussion. Three papers relevant to Item 1 were discussed [1-3]; one for Item 2 [4]; and two for Item $4[5,6]$. An additional paper for Item $2[7]$ arrived too late for discussion.

Two notes on terminology: first, the theme "Sex and Gender in Health and Medicine" signals a distinction between "sex" and "gender". Some social scientists distinguish male-female behaviors and differences due to biology (sex) from those due to social roles (gender). Throughout the Conference, the Discussion Group used the word "sex" in an inclusive sense. Thus, the term "sex differences" referred to male-female differences due to biological and/or social factors. Consistent with Conference practice, this report uses "sex" in a global sense. Second, in this report the words "male" and "female" refer to persons of any age; "men" and "women", to adults.

Following is a summary of the ideas and issues covered by the Discussion Group:

\section{SEX DIFFERENCES IN MORBIDITY. MORTALITY, AND UTILIZATION}

\section{Papers}

Nathanson's paper on "Sex, Illness, and Medical Care: a Review of Data, Theory, and Method" [1] is a Background Paper for the Conference. It reviews 
international data on sex differences in illness and medical care, compares various health indicators, comments on reporting errors in health interviews, and considers possible reasons for the sex differences presented. Verbrugge's paper "Sex Differences in Morbidity and Mortality in the United States" [2] asks why health interviews show higher morbidity for females than males, but death rates are higher for males. A second paper "Females and Illness: Recent Trends in Sex Differences in the United States" [3] presents trends in sex differences for morbidity and disability for the period 1957-1972, and compares them with mortality trends for the same period. Empirical evidence suggests that excess female morbidity and disability are due primarily to social and psychological factors which influence survey reports.

\section{Discussion}

The group reviewed evidence on sex differences in morbidity and mortality. Throughout the world, males have higher death rates than females at all ages. There are few exceptions to this statement. How much males' excess mortality is due to biological factors, versus social ones, is not known. There is some support for the biological argument: females of many animal species outlive males. Among humans, fetal death rates are higher for males than females. But social factors are also important: in most societies, males are exposed to more health hazards than females, due to their occupations, leisure activities, and life styles. Which aspects of males' lives lead to excess mortality are not known, but research points to smoking as a major factor.

Health interview data generally show higher morbidity, disability, and utilization among females than males. This is especially true for acute conditions, but it is also apparent for some chronic ones. The female excess may be due to more physical morbidity. But it is plausible that females are more sensitive to symptoms, more willing to admit symptoms, and more able to take time for disability and medical care, compared to males. In summary, socialization about health may account for the higher female rates, rather than higher physical morbidity.

The causes of illness and death vary for developed and developing countries. This means that the size of sex differences and the relative importance of their causes also vary. Mortality data are available for numerous countries, and sex mortality differences have been examined [8]. Health data are more restricted to developed countries, and most data refer to utilization or disability. Analysis of such data is limited to reporting the direction of sex differences but not exploring the relative size of those differences $[1,9]$.

A group member commented that non-developed (primitive) societies may show very different patterns in male and female health. Anthropological evidence indicates that male children are favored throughout the world. This may cause higher use of traditional services for boys than girls (and for men than women) in primitive settings.

Several other topics were discussed:

(a) $A$ "rhetoric of health"

How people describe their symptoms probably varies by sex, age, and socio-economic characteristics. Females may use different words for a symptom than males do, or give more elaborate details about their discomforts. Little is known about this rhetoric of health.

\section{(b) Legitimacy of the sick role}

Females may use illness as an excuse to avoid work, school, or other commitments more than males do. Does the public accept "sickness" of girls and women, more than of boys and men? Does this acceptance vary among countries?

\section{(c) Data sources and perspectives of health}

Prominent sources of national and international health data were named. Differences in the health items included were discussed: (i) Many studies confound morbidity and utilization, by computing incidence and prevalence rates based solely on conditions which prompted someone to seek medical care. (ii) Some data on illness conditions are diagnostic; others are self-reports. Diagnoses of conditions fit a medical perspective of illness. Self-reports of conditions fit a social perspective, which claims that a person is sick if he or she feels sick. Both perspectives are important, and the group agreed that medical data should not be viewed as more valid than self-report data. (Some members noted a congruence between the social perspective of illness and the "self-help" health movement. In "self-help" settings, an individual's entire health and well-being is considered, rather than just a specific disease or complaint.) (iii) The group also compared the notions of "illness" and "fitness". In summary, group members agreed that sources of health data should be examined closely for sex differences, and that new data aimed at specific hypotheses should be collected.

\section{MEN AND WOMEN AS HEALTH PROFESSIONALS}

\section{Papers}

Levitt's paper on "Men and Women as Providers of Health Care" [7] is a Background Paper for the Conference. It discusses the sex composition of medical professions in the United States and argues that "scientific medicine" legitimates male domination of high-prestige health jobs. Segovia and Elinson's [4] paper described how male and female physicians in Buenos Aires differ in medical specialties, income sources, and professional attitudes. Compared to men, women physicians are especially likely to be pediatricians, psychiatrists, and gynecologists. They are unlikely to be surgeons or in general medicine. Women physicians appear to have non-academic (low prestige) positions more than men physicians. A larger share of the women's income comes from salaries (rather than fees), and more of the women consider their incomes below average. More often than their female peers, male physicians think it is desirable for a doctor to be the same sex as the patient, and that the quality of medical care is better in that situation. Male physicians also think that having the same religion and same national origin are important in doctor-patient interaction, more than female physicians do. 


\section{Discussion}

The group considered two topics: how men and women are recruited to health professions, and if a professional's sex influences his/her skills, attitudes, and behavior.

The predominance of males as physicians and females as nurses was attributed to social institutions and public expectations. In most countries, pre-medical and medical training programs have long welcomed men students more than women students. Conversely, nursing training has been aimed at women. There are strong socialization factors that support these institutional filters: children learn that "doctors are men, and nurses are women". This influences their career dreams and deters then from wanting to enter some health professions. Explicit advice from parents and teachers also affects their plans. (For example, parents may caution a son that male nurses are considered homosexuals and urge him to avoid a profession which might cause him embarrassment.)

All countries have norms (expectations) for the sex of health professionals, but these norms vary. In other words, there are always institutional and attitudinal filters focused on sex, but the filters vary throughout the world. The historical and cultural reasons for these filters merit research.

Some group members stated that institutional and attitudinal factors do affect professional recruitment, but that males and females still are not equally suited to all health professions. Given equal opportunities, men would still tend to become physicians and women, nurses, because of biological factors. Maternal instincts and other nurturant qualities would urge females toward the health professions which involve close personal contact with patients. Even if they become physicians, women will behave differently from men physicians, empathizing more with clients and treating them in a more holistic fashion. Similarly, whether they are nurses or physicians, men will focus on the treatment of specific disease conditions and limit their emotional involvement with patients. There was lively discussion of this biological argument by the group.

In summary, members agreed that social factors are important in how the sexes are recruited to health professions, but they disagreed about the relative importance of biological factors.

\section{NON-MARKET AND TRADITIONAL HEALTH SERVICES}

(Non-market health services received little attention during the Conference by the Sex and Gender group. Traditional health services and practitioners were discussed in the Primary Health Care group, but not by the Sex and Gender group.)

\section{SEX-SPECIFIC HEALTH CONDITIONS}

Papers

Sidenius' paper "Study of Women Seeking Abortion" [6] presents trends in abortion rates for Denmark and outlines a proposed study of women who have obtained abortions. The study will concentrate on how the unwanted pregnancy occurred and how the abortion decision was made. A second paper titled "Control of the Emotions, Remote Stress, and the Emergence of Breast Cancer" [5] considers how stress and emotional control may influence cancer development. The paper reports data for women admitted to a hospital for breast tumor biopsy. The Sex and Gender group chose to discuss the paper because of the cancer site and because of members' interest in the topic of stress and illness. Bagley finds that women who repress their emotions and had severe stress many years before the biopsy are especially likely to have positive diagnoses of breast cancer. Women who report no "old" stress and tend to express their emotions (rather than "bottle them up") are least likely to have that diagnosis. Considering predictor variables separately, these are associated with a positive diagnosis: recent stress, "old" stress, and emotional repression.

\section{Discussion}

Two topics received extensive attention: reproductive decision-making and the relationship of stress to illness.

(a) Reproductive decision-making. Recent trends in abortion and birth rates in Denmark were compared with rates for other developed countries. Increased abortion rates in Denmark are related to liberalization of abortion laws there (the most recent revision occurring in 1973). From aggregate rates and other data on contraceptive sales and prescriptions, some inferences about reproductive decisions are possible. But to understand fully how women and their male partners choose birth prevention methods and decide to have children requires detailed responses from women and men. Some Danish researchers are curious about whether liberal abortion and contraception laws reduce men's feelings of responsibility for reproduction, so they assume that women are contracepting and insist on abortion if a pregnancy occurs. This hypothesis will be investigated in the proposed Danish study. In general, processes of reproductive decision-making merit careful study throughout the world. The group applauded the work of the World Fertility Study, which provides comparative data on fertility and fertility regulation for numerous countries of the world.

(b) Stress and illness. Bagley's paper prompted discussion of (i) the design of studies for research on stress and illness and (ii) the influence of hormones on psychological and physical symptoms.

(i) Several persistent problems occur in research on stress and illness. First, if an association between them is found, it may not be easily interpreted in a causal sense. Usually, researchers wish to demonstrate that stress influences (or does not influence) illness. But an association based on retrospective data can be due to the opposite effect (illness causes stress): people's psychological characteristics are queried or tested at the time of, or after, the medical diagnosis. Even if they are not aware of the diagnosis when the psychological measurements occur, people may have sufficient clues and information to make a good guess. "Foreknowledge" of diagnosis undoubtedly influences patients' moods and feelings of stress, and the psychological data reflect that. To avoid this problem, pro- 
spective studies are recommended, since psychological measures are made before the occurrence of disease. Relationships between stress and illness can then be interpreted in a causal manner with more confidence. The disadvantages of prospective studies are their high cost and sample attrition over time.

Second, a determined effort must be made to understand how stress influences illness, if an association is found. The hypothesis that high stress makes people vulnerable to acute and chronic health problems has intrigued scientists for several decades. It has been a central premise of psychosomatic medicine, and it is currently exciting research by social epidemiologists. High stress may increase illness and injury by encouraging high-risk life styles or by triggering biochemical processes that result in illness. Group members agreed that social scientists who investigate stress-illness relationships should be assisted by epidemiologists, who can inform them about the etiology of diseases being studied. Intervening processes between stress and illness should be understood and, if possible, measured in current investigations, rather than be assumed.

Third, the group noted that "stress" can be operationalized in numerous ways. Inconsistent empirical results may sometimes be due to different definitions of operational indicators of stress. The group commented that researchers must identify clearly how stress is conceptualized and measured in empirical studies.

(ii) How hormones influence emotions, behavior and illness in both sexes is an intriguing issue. For example, some women are particularly depressed and feel ill just before their menses. This has been labelled the "premenstrual syndrome". A popular explanation is that it is caused by cyclic fluctuations in progesterone. (An alternative explanation is strictly socialpsychological: that some women who dislike menstruation become depressed and upset several days beforehand.) With respect to Bagley's research, group members suggested that breast cancer may be hormone-dependent, and that the stress-cancer relationship is a spurious one. In other words, women with particular hormone profiles may become prone to both stress and breast cancer.

The group agreed on a general caution for research on stress and illness: social scientists should consider the possibility of spurious relationships (i.e. some factor causes both stress and illness). If spurious results are possible, researchers should either design their studies to test them, or acknowledge their possibility and cite available evidence in data analysis.

\section{SEX BIASES IN MEDICAL CARE AND PUBLIC EXPECTATIONS}

The notion of sex biases arose several times during the group discussions, and participants clearly believed that such biases exist even if they are poorly documented by research.

\section{Discussion}

(a) Sex biases in medical care. Several members argued strongly that physicians overprescribe medications and advice unnecessary surgery for their women patients. Recent data on surgery in the United States show increased rates for most surgical procedures, but especially striking increases for hysterectomy. In general, increased surgery rates may reflect needed surgery for people now covered by Medicare and Medicaid programs. Alternatively, they reflect unnecessary surgery advised by physicians who can obtain income readily from the government for the procedures. There is suspicion that high hysterectomy rates are partly due to the latter.

Female patients are usually treated by male physicians. Traditional sex and doctor-patient roles give the physician power in these situations, but it is thought to be especially likely in gynecological/obstetric practice. Women's liberation groups have been concerned about "exploitation" of females by male physicians. One result is the emergence of self-help and women's health clinics.

(b) Sex biases in public expectations. The claim above is that physicians treat patients differentially according to the patient's sex. It is also possible that patients react to doctors according to the doctor's sex. Patients hold expectations about the sex of health professionals: they expect authority from physicians, and empathic care from nurses. Traditionally, these are (respectively) male and female characteristics. When contacting a professional whose sex is "unusual", patients may express surprise and feel less satisfied with the care received. These patient responses, plus incomplete acceptance by colleagues, may embarrass the "unusual" professionals and influence their behavior with clients and peers.

\section{CONCLUSION}

The importance of sex in health experiences and mortality, medical treatment, and the structure of medical care is as great in developing countries as in developed ones. Members of the Sex and Gender group all came from developed countries, and the specific topics discussed reflected that similar origin. People from developing countries would have contributed greatly to discussions of traditional medicine, sex mortality differentials and their causes, and sex composition of the health professions.

The Sex and Gender group drafted no specific policy or research recommendations. However, there was agreement on these points:

(1) Sex differences in health (morbidity, disability, utilization of health services) merit more research, on existing and new data.

(2) Males and females should have equal opportunities to train and practice any health profession. In addition to reducing institutional impediments to training, the lay public should be socialized to accept health professionals regardless of their sex.

(3) Reproductive decision-making and the relationship between stress and illness are not well understood and deserve active research attention by social scientists. Work on the second topic should be interdisciplinary, including epidemiologists familiar with the disease(s) being studied.

(4) Despite widespread belief that sex biases by physicians exist, there is little empirical demonstration of them. These biases are especially difficult to study in a quantitative fashion, since medical professionals may resist having their decisions and 
motives examined. Such data are necessary, however, to verify (or discount) sex biases.

\section{REFERENCES}

1. Nathanson C. A. Sex, illness, and medical care: a review of data, theory, and method. Soc. Sci. Med. 11, 13, 1977.

2. Verbrugge L. M. Sex differentials in morbidity and mortality in the United States. Soc. Biol. 23, 275, 1976.

3. Verbrugge L. M. Females and illness: recent trends in sex differences in the United States. J. Hith Soc. Behav. 17, 387, 1976.

4. Segovia J. and Elinson J. Sex differences in medical practice in Argentina. Paper presented at the Fifth International Conference on Social Science and Medicine, 1977. (First author address: Division of Community Medicine, Memorial University of Newfoundland. St. John's. Newfoundland, Canada A1B 3V6.)
5. Bagley C. Control of the emotions, remote stress, and the emergence of breast cancer. Paper presented at the Fifth International Conference on Social Science and Medicine, 1977. (Department of Sociology, University of Surrey, Guildford, U.K.)

6. Sidenius K. Study of women seeking abortion. Paper preeented at the Fifth International Conference on Social Science and Medicine, 1977. (Institute of Social Medicine, University of Copenhagen, 32 Juliane Mariesvej, DK 2100 Copenhagen, Denmark.)

7. Levitt J. Men and women as providers of health care. Soc. Sci. Med. 11, 395, 1977.

8. Preston S. H. and Weed J. A. Causes of death responsible for international and intertemporal variation in sex mortality differentials. World Hlth Statist. Rept 29(3), 144, 1976.

9. Kohn R. and White K. Health Care: An International Study. Oxford University Press, London, 1978. 